\title{
A correlate of protection for SARS-CoV-2 vaccines is urgently needed
}

\author{
Recent studies suggest that neutralizing antibodies could serve as a correlate of protection for vaccines against \\ SARS-CoV-2 in humans.
}

\section{Florian Krammer}

$\Lambda$ daptive humoral and cellular immune responses to infection are typically pathogen specific and protect against future reinfection. In vaccines, science has found a way for humans to acquire these protective immune responses without suffering an initial infection. Vaccine-induced immune responses are often multifaceted, but single components such as antibody responses may correlate with the level of protection. In fact, most of the currently accepted correlates of protection are based on antibody measurements ${ }^{1,2}$. In many cases they may not be the only correlate of protection, but they are often much easier to measure than cellular responses and are therefore more clinically useful. Notably, correlates may differ depending on the endpoint used, such as protection from infection, from disease, from severe disease or from mortality. Two studies, by Khoury et al. ${ }^{3}$ (published in this issue of Nature Medicine) and Earle et al. ${ }^{4}$, now connect neutralizing antibody responses to SARS-CoV-2 with vaccine efficacy and bring researchers and clinicians closer to having a correlate of protection for vaccines against COVID-19.

To place these studies in context, it is important to first understand why a correlate of protection and, more specifically, an 'absolute correlate' (meaning a protective threshold) ${ }^{2}$ are urgently needed. First, more vaccines are needed for global protection against SARS-CoV-2, particularly in low- and middle-income countries. The currently licensed vaccines cannot be produced in large enough quantities to satisfy this need in a timely manner; therefore, more vaccines need to be licensed. Many candidates are in advanced stages of clinical development, but conducting phase 3 efficacy studies is challenging due to ethical reasons (the need for non-inferiority trials with a standard-of-care comparator instead of a placebo) and due to a reduction in the number of cases (which means trials may take longer or may require a larger number of participants). Many of

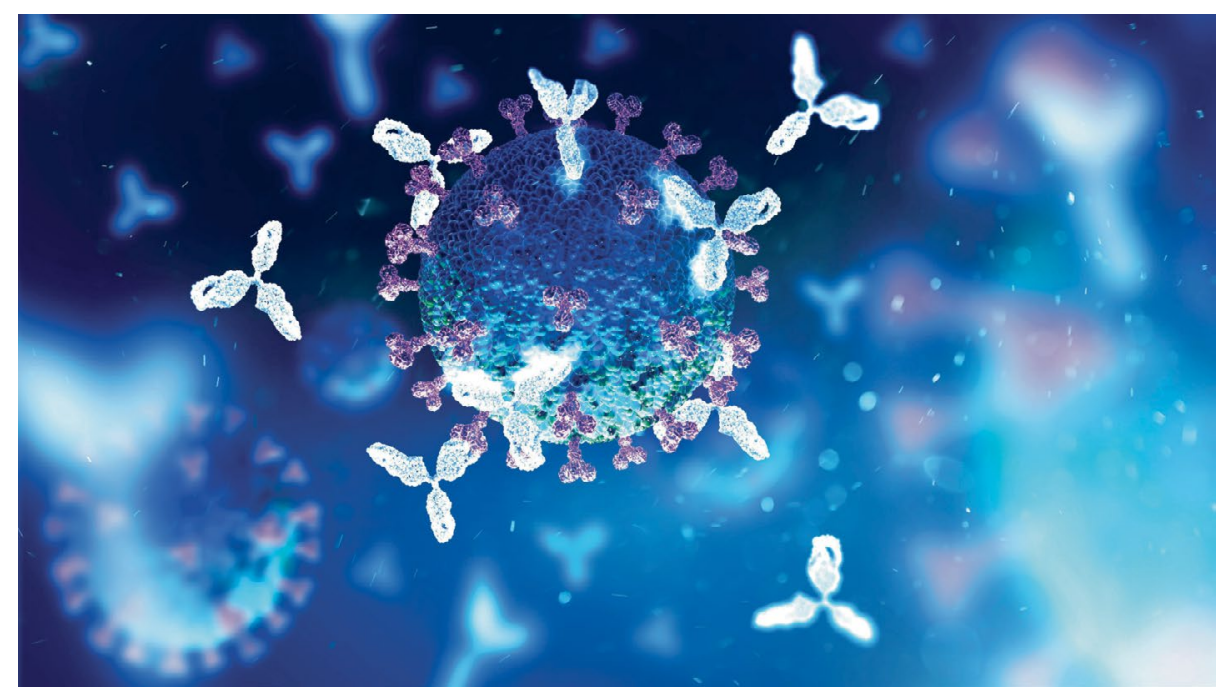

Credit: Andrew Vodolazhskyi / Alamy Stock Photo

the vaccine candidates that are still in the pipeline are being developed by smaller companies or producers in low- and middle-income countries who may not be able to conduct the large phase 3 trials needed to get their products licensed. Having a correlate of protection that allows licensure based on immune readouts would circumvent these issues and would undoubtedly boost vaccine availability.

Many individuals with a compromised immune system, including transplant patients, patients with cancer and individuals taking immunosuppressive drugs, do not mount strong responses to vaccines, including those for SARS-CoV-2 $2^{5-8}$. At present, the messaging from regulatory agencies states that "antibody tests should not be used to evaluate a person's level of immunity or protection from COVID-19." If there is no detectable antibody response after vaccination, the vaccines may still offer protection through cellular immunity. Although this may be true in some cases, cellular immune responses and antibody responses often correlate to some extent ${ }^{10}$.
Mechanistically, the induction of strong antibody responses is dependent on $\mathrm{CD} 4^{+}$ $\mathrm{T}$ cell responses, and there are preliminary reports of immunocompromised individuals who did not mount antibody responses to vaccination against SARS-CoV-2 and subsequently succumbed to infection ${ }^{5}$. Having an established correlate of protection would allow healthcare providers to manage the vaccination of immunocompromised individuals more efficiently, such as by recommending booster vaccinations if antibody titers are too low, or recommending non-pharmaceutical interventions for protection if no immune response is detected.

Having a correlate of protection would also allow healthcare and governing bodies to efficiently determine what percentage of the population is protected. Although seroprevalence is currently used as a crude measure of community immunity, having a correlate of protection would allow more-precise estimations that could then trigger interventions such as vaccination campaigns if the percentage of immune individuals is deemed to be too low. 
The studies by Khoury et al. ${ }^{3}$ and Earle et al. ${ }^{4}$ take a first and important step toward a correlate of protection. The authors used data from published clinical studies of several vaccines against SARS-CoV-2 and evaluated the relationship between efficacy and neutralizing antibody titers. This was not straightforward, because neutralization assays differed vastly among the studies analyzed by both teams, and an international standard to facilitate direct cross-comparison was not available until late in 2020 ( ref. $^{11}$ ). However, many of the studies used panels of convalescent serum as a comparator for vaccine-induced antibody titers, so the authors normalized the neutralization titers of vaccinees to those of convalescent people. Although it is not without its limitations, this approach allowed direct comparison between studies of 'normalized' neutralization titers. The results from both teams showed a significant correlation between vaccine efficacy and vaccine-induced neutralizing antibody activity. Even titers of binding antibody (e.g., as measured by enzyme-linked immunosorbent assays, which are much easier to perform at large scale than neutralization assays are) seemed to correlate well with efficacy ${ }^{4}$. These findings suggest that antibodies may provide a correlate of protection, with further support for this coming from animal studies ${ }^{12}$ and natural-infection cohorts ${ }^{13}$.

Relying on a correlate of protectionalthough extremely helpful in many waysalso comes with certain risks. Although certain correlates can be non-mechanistic ${ }^{1}$, meaning an immune marker that indicates protection but does not cause it, antibodies are often mechanistic correlates ${ }^{1}$ of protection, especially if they are capable of neutralizing the pathogen in question. Correlates may be specific to a vaccine platform or even specific to a vaccine. Some vaccines may be highly protective but may not induce the type of immunity established as a correlate and, vice versa, a vaccine may induce the immune response used as correlate but may still not provide protection, especially when a non-mechanistic correlate of protection is used. Nevertheless, robust preclinical and clinical studies make these scenarios unlikely; vaccine developers understand the type of immunity their vaccines induce, and animal experiments ${ }^{12}$ (and monoclonal antibody therapeutics in humans) show that antibodies do directly participate in protection (and therefore are probably a mechanistic correlate of protection $)^{10}$.

A more robust threshold of protection, based on data from individual people instead of pooled efficacy data, can be derived from breakthrough cases in phase 3 studies and observational studies. Therefore, swift data sharing and collaboration to establish an absolute correlate of protection should be the number one priority for vaccine producers, academic researchers and regulatory agencies. Although it is unlikely that such an effort will arrive at a flawless absolute correlate that can be applied to all vaccine candidates, all viral variants and all patient populations, it would certainly be extremely helpful in catalyzing the licensure of more vaccines, guiding patient management and informing public-health decisions.

\section{Florian Krammer (ID) 1,2区}

${ }^{1}$ Department of Microbiology, Icahn School of Medicine at Mount Sinai, New York, NY, USA.
${ }^{2}$ Department of Pathology, Icahn School of Medicine at Mount Sinai, New York, NY, USA.

$\bigotimes_{\text {e-mail: florian.krammer@mssm.edu }}$

Published online: 8 July 2021

https://doi.org/10.1038/s41591-021-01432-4

References

1. Plotkin, S. A. \& Gilbert, P. B. Clin. Infect. Dis. 54, 1615-1617 (2012).

2. Plotkin, S. A. Clin. Vaccine Immunol. 17, 1055-1065 (2010).

3. Khoury, D.S. et al. Nat. Med. https://doi.org/10.1038/s41591-02101377-8 (2021).

4. Earle, K.A., et al. Vaccine https://doi.org/10.1016/j.vaccine. 2021.05.063 (2021).

5. Aleman, A., et al.. Preprint at medRxiv https://doi.org/10.1101/ 2021.05.15.21256814 (2021).

6. Boyarsky, B. J. et al. J. Am. Med. Assoc. 325, 1784-1786 (2021).

7. Agha, M., Blake, M., Chilleo, C., Wells, A. \& Haidar, G. Preprint at medRxiv https://doi.org/10.1101/2021.04.06.21254949 (2021).

8. Deepak, P., et al. Preprint at medRxiv https://doi. org/10.1101/2021.04.05.21254656 (2021).

9. U.S. Food \& Drug Administration. https://www.fda.gov/medicaldevices/safety-communications/antibody-testing-not-currentlyrecommended-assess-immunity-after-covid-19-vaccinationfda-safety (2021).

10. Grifoni, A. et al. Cell 181, 1489-1501.e1415 (2020).

11. Kristiansen, P. A. et al. Lancet 397, 1347-1348 (2021).

12. McMahan, K. et al. Nature 590, 630-634 (2021).

13. Krammer, F. Lancet 397, 1421-1423 (2021).

\section{Acknowledgements}

Work on SARS-CoV-2 in the Krammer laboratory is funded by the NIAID Collaborative Influenza Vaccine Innovation Centers (contract 75N93019C00051), NIAID Center of Excellence for Influenza Research and Surveillance (contract HHSN272201400008C), by the generous support of the JPB Foundation and the Open Philanthropy Project (research grant 2020-215611 (5384)), and by anonymous donors.

\section{Competing interests}

The Icahn School of Medicine at Mount Sinai has filed patent applications relating to serological assays for SARS$\mathrm{CoV}-2$ and Newcastle disease virus-based vaccines against SARS-CoV-2 that list F.K. as co-inventor. Mount Sinai has 'spun out' a company, Kantaro, to market serological tests for SARS-CoV-2. F.K. has consulted for Merck and Pfizer (before 2020) and currently consults for Pfizer, Seqirus and Avimex. The Krammer laboratory is also collaborating with Pfizer on animal models of SARS-CoV-2.

\section{Dominantly inherited Alzheimer's disease: a compass for drug development}

The first phase 3 trial of amyloid- $\beta$-targeting monoclonal antibodies in dominantly inherited Alzheimer's disease failed to slow cognitive decline in patients. Could it still help to inform future study design and drug development in this setting?

\section{Gil D. Rabinovici}

ominantly inherited Alzheimer's disease (DIAD) accounts for only $1 \%$ of cases of Alzheimer's disease (AD) but has made fundamental contributions to the understanding of this disease ${ }^{1}$. The discovery in the 1990s that familial early-onset $\mathrm{AD}$ is caused by mutations in one of three genes encoding amyloid precursor protein (APP), presenilin-1 or presenilin-2, all of which promote the cleavage of APP into amyloidogenic 40- or 42 -amino acid polypeptides $\left(\mathrm{A} \beta_{40}\right.$ and $\left.\mathrm{A} \beta_{42}\right)$, 